\title{
Algorithms and Properties for Positive Symmetrizable Matrices
}

\author{
E.S. DIAS ${ }^{1 *}$, D. CASTONGUAY ${ }^{1}$ and M.C. DOURADO ${ }^{2}$
}

Received on November 24, 2015 / Accepted on July 24, 2016

\begin{abstract}
Matrices are one of the most common representations of graphs. They are also used for representing algebras and cluster algebras. A symmetrizable matrix $M$ is one for which there is a diagonal matrix $\mathrm{D}$ with positive entries, called symmetrizer matrix, such that $D M$ is symmetric. This paper provides some properties of matrices in order to facilitate the understanding of symmetrizable matrices with specific characteristics, called positive quasi-Cartan companion matrices, and the problem of localizing them. We sharpen known coefficient limits for such matrices. By generalizing Sylvester's criterion for symmetrizable matrices we show that the localization problem is in NP and conjectured that it is NP-complete.
\end{abstract}

Keywords: symmetrizable matrix, positive quasi-Cartan matrix, algorithm.

\section{INTRODUCTION}

Matrices can be used to represent various structures, including graphs and algebras. For instance, a cluster algebra can be defined using a directed graph, called quiver, and consequently by an adjacency matrix, where rows and columns represent the vertices and the positive values at positions $(i, j)$ represent the number of edges between associated vertices of the graph. For more information about quiver and cluster algebras, see [1, 6, 7].

Cartan matrices were introduced by the French mathematician Élie Cartan [2]. In fact, Cartan matrices, in the context of Lie algebras, were first investigated by Wilhelm Killing [8], whereas the Killing form is due to Cartan.

The notion of quasi-Cartan matrices was introduced by Barot, Geiss and Zelevinsky [1] who reported some their properties. Those matrices are symmetrizable and when associated with a skew-symmetrizable matrix are called a quasi-Cartan companion. In Section 4, we sharpen the bounds on the entries of a positive quasi-Cartan matrices, given by these authors.

\footnotetext{
*Corresponding author: Elisângela Silva Dias.

${ }^{1}$ Instituto de Informática, Universidade Federal de Goiás, Alameda Palmeiras, Quadra D, Campus Samambaia, 74001-970 Goiânia, GO, Brasil. E-mails: elisangela@inf.ufg.br; diane@inf.ufg.br

2 Departamento de Ciência da Computação, Instituto de Matemática, Universidade Federal do Rio de Janeiro, Av. Athos da Silveira Ramos, 274, Ilha do Fundão, 21941-916 Rio de Janeiro, RJ, Brasil. E-mail: mitre@dcc.ufrj.br
} 
By Sylvester's criterion [3], a symmetric matrix is positive semidefinite if all its leading principal submatrices have positive determinants. A symmetrizable matrix is positive if its associated symmetric matrix is also positive. In Section 4 we generalize Sylvester's criterion for symmetrizable matrices.

Furthermore, one can decide whether a cluster algebra is of finite type (has a finite number of cluster variables) by deciding whether the associated matrix has a positive quasi-Cartan companion matrix. There are also other decision criteria, but they will not be discussed in the present paper.

In this paper, we study the quasi-Cartan matrices from a mathematical and computational point of view. Section 2 is devoted to some notations and definitions. In Section 3, we first present some properties that a matrix should have in order to be a quasi-Cartan companion of a skewsymmetrizable matrix $B$. After this, we developed two algorithms: the first one decides in time complexity $\Theta\left(n^{2}\right)$ if the matrix is symmetrizable and returns the symmetrizer if it exists; the second one finds a symmetrizer matrix for a symmetrizable matrix, having time complexity $\Theta\left(n^{2}\right)$ in the worst case and $\Theta(n)$ in the best case. In Section 4, via generalization of Sylvester's criterion for symmetrizable matrices, we conclude that the problem of deciding if a matrix has a positive quasi-Cartan companion is in NP. A problem belongs to NP class of problems if there exists a non-deterministic Turing machine of a unique tape that decides it in polynomial time, that is, problems in NP are those can be verified in polynomial time. Since there is an exponential number of quasi-Cartan companions, we conjecture that the decision problem is in NP-complete. We also sharpen the bounds on the entries of a positive quasi-Cartan matrix given by Barot, Geiss and Zelevinsky [1] and highlight specific cases where we always have a positive quasi-Cartan companion or where one can easily verify this fact. The paper ends with our conclusions in Section 5 .

\section{PRELIMINARIES}

In this paper, we consider square matrices with integer entries, except the matrix $D$ that will be introduced later. Let $n$ be a positive integer, $A, B, C \in \mathrm{M}_{n}(\mathbb{Z})$ and $D \in \mathrm{M}_{n}(\mathbb{R})$. A matrix $A$ is symmetric if $A=A^{T}$, where $A^{T}$ is the transpose of $A$. A matrix $C$ is symmetric by signs if for all $i, j \in\{1, \ldots, n\}$, with $i \neq j$, we have $c_{i j}=c_{j i}=0$ or $c_{i j} \cdot c_{j i}>0$. A matrix $C$ is symmetrizable if $D C$ is symmetric for some diagonal matrix $D$ with positive diagonal entries. In this case, the matrix $D C$ is called symmetrization or symmetrized of $C$ and the matrix $D$ is called symmetrizer of $C$. Note that this definition of symmetrizable is equivalent to the one given in [4] and that it is not unique.

The matrix $A$ is termed skew-symmetric if its transpose coincides with its opposite $\left(A^{T}=-A\right)$, i.e., $a_{i j}=-a_{j i}$, for all $i, j$. Observe that the values of the main diagonal are null. The matrix $B$ is termed skew-symmetric by signs if for all $i, j \in\{1, \ldots, n\}$ we have $b_{i i}=0$ and if $i \neq j$, then $b_{i j}=b_{j i}=0$ or $b_{i j} \cdot b_{j i}<0$. The matrix $B$ is skew-symmetrizable if there exists a diagonal matrix $D$ with positive entries such that $D B$ is a skew-symmetric matrix. In this case, the matrix $D B$ is called askew-symmetrization or skew-symmetrized of $B$ and the matrix $D$ is called a skew-symmetrizer of $B$. 
We observe that every symmetric matrix is symmetrizable and that every skew-symmetric matrix is skew-symmetrizable. Also observe that all symmetrizable matrices are symmetric by signs and all skew-symmetrizable matrices are skew-symmetric by signs.

A symmetrizable matrix is quasi-Cartan if all the entries of its main diagonal are equal to 2 . For a skew-symmetrizable matrix $B$, we shall refer to a quasi-Cartan matrix $C$ with $\left|c_{i j}\right|=\left|b_{i j}\right|$ for all $i \neq j$ as a quasi-Cartan companion of $B$.

Given a skew-symmetrizable matrix $B$, we want to find a positive quasi-Cartan companion of $B$. For this, we use Sylvester's criterion and need the following notations and definitions.

For any matrix $A$, we denote as $A_{[i j]}$ the matrix obtained by the elimination of the $i^{t h}$ row and the $j^{\text {th }}$ column of matrix $A$. The $i j^{\text {th }}$ minor of $A$ is the determinant of $A_{[i j]}$. Recall that the determinant of $A$ can be recursively defined in terms of its minors. More generally, a principal submatrix of $A$ is a submatrix of $A$ obtained by eliminating some rows and respective columns of $A$. The principal minors of $A$ are the determinants of the principal submatrices of $A$. The leading principal minors are the determinants of the diagonal blocks of a matrix $M$ with dimension $1,2, \ldots, n$. These submatrices, also called leading principal matrices, are obtained by eliminating the last $k$ columns and $k$ rows of $A$, with $k=n-1, n-2, \ldots, 0$.

In Section 4, we shall use the concept of a connected matrix defined below.

For any arbitrary matrices $A$ of dimension $m \times n$ and $B$ of dimension $p \times q$, we define the direct sum of $A$ and $B$, denoted by

$$
A \oplus B=\left[\begin{array}{ll}
A & 0 \\
0 & B
\end{array}\right] .
$$

Note that any element in the direct sum of two vector spaces of matrices can be represented as the direct sum of two matrices.

A permutation matrix is a square binary matrix that has exactly one unit entry in each row and each column and zeros elsewhere. Each such matrix represents a specific permutation of $n$ elements and, when used to multiply another matrix, it will produce a permutation of the rows or columns of the other matrix.

Let $A$ be a square matrix. We say that $A$ is disconnected if there exists $P$, a permutation matrix, such that $P A P$ is a direct sum of at least two non-zero matrices. If not, we say $A$ is connected. Observe that $P A P$ is obtained from $A$ by a permutation of the rows and respective columns of $A$. Moreover, $A$ is connected if, and only if, the graph associated with the adjacency matrix $A$ is connected. More information on the properties of matrices is given in [9].

\section{SYMMETRIZABLE AND SKEW-SYMMETRIZABLE MATRICES}

In this section, we report some properties of symmetrizable and skew-symmetrizable matrices. We also present two algorithms for symmetrizable matrix. 
The following proposition demonstrates that all possible symmetric by signs matrices are quasiCartan companions and thus, there is an exponential number of such matrices since a quasiCartan companion of a matrix $B$ is specified by choosing the signs of its off-diagonal matrix entries, with the only requirement being that $\operatorname{sgn}\left(c_{i j}\right)=\operatorname{sgn}\left(c_{j i}\right)$ and $\left|c_{i j}\right|=\left|b_{i j}\right|$ for all $i \neq j$.

Theorem 3.1. Let $B$ be a skew-symmetrizable matrix. Consider a matrix $C$ such that $\left|c_{i j}\right|=\left|b_{i j}\right|$, for all $i \neq j$. If $C$ is symmetric by signs, then $C$ is symmetrizable with the same symmetrizer as that of $B$. Furthermore, if $c_{i i}=2$, for all $i$, then $C$ is a quasi-Cartan companion of $B$.

Proof. Let

$$
D=\left(\begin{array}{ccc}
d_{1} & & \\
& \ddots & \\
& & d_{n}
\end{array}\right)
$$

be a symmetrizer matrix of $B$. Then we have that $d_{i} \cdot\left|c_{i j}\right|=d_{i} \cdot\left|b_{i j}\right|=\left|d_{i} \cdot b_{i j}\right|=\left|d_{j} \cdot b_{j i}\right|=$ $d_{j} \cdot\left|b_{j i}\right|=d_{j} \cdot\left|c_{j i}\right|$. Since $c_{i j}$ and $c_{j i}$ have the same sign, we have that $d_{i} \cdot c_{i j}=d_{j} \cdot c_{j i}$.

Next result for symmetrizable matrices is similar to the result in [7](Lemma 7.4) for skewsymmetrizable matrices.

Lemma 3.1. A matrix $C=\left(c_{i j}\right)$, of order $n$, is symmetrizable if and only if it is symmetric by signs and for all $k \geq 3$ and all $i_{1}, i_{2}, \ldots, i_{k} \in\{1, \ldots n\}$ it satisfies:

$$
c_{i_{1} i_{2}} \cdot c_{i_{2} i_{3}} \cdot \ldots \cdot c_{i_{k} i_{1}}=c_{i_{2} i_{1}} \cdot c_{i_{3} i_{2}} \cdot \ldots \cdot c_{i_{1} i_{k}} \cdot
$$

We now present two algorithms: one for deciding whether any given square matrix of order $n$ has a symmetrizer and, if it exists, returns it with time complexity $\Theta\left(n^{2}\right)$. Another one for finding symmetrizer matrix for a symmetrizable matrix with time complexity $\Theta\left(n^{2}\right)$ in the worst case and $\Theta(n)$ in the best case. Since the first algorithm has time complexity $\Theta\left(n^{2}\right)$ if we know that a given matrix is symmetrizable using Algorithm 2 is more suitable than Algorithm 1.

Observe that we do not have a unique symmetrizer for a symmetrizable matrix. For the sake of simplicity, we shall calculate a symmetrizer with positive values and rational entries.

It is important to observe that there exist algorithms that use breadth first strategy to decide whether or not there exists a symmetrizer matrix or not. Our algorithm solves the same problem and presents such symmetrizer.

Note that if $A$ is connected, then we effectuate line 8 only once.

Proposition 3.1. Algorithm 1 is correct.

Proof. At the beginning of any iteration of the "while loop", $d_{i i} \cdot a_{i j}=d_{j j} \cdot a_{j i}$ for any $j \notin T$ and any $i$. This is clearly true since at the beginning of the algorithm there is no $j$ and afterwards 


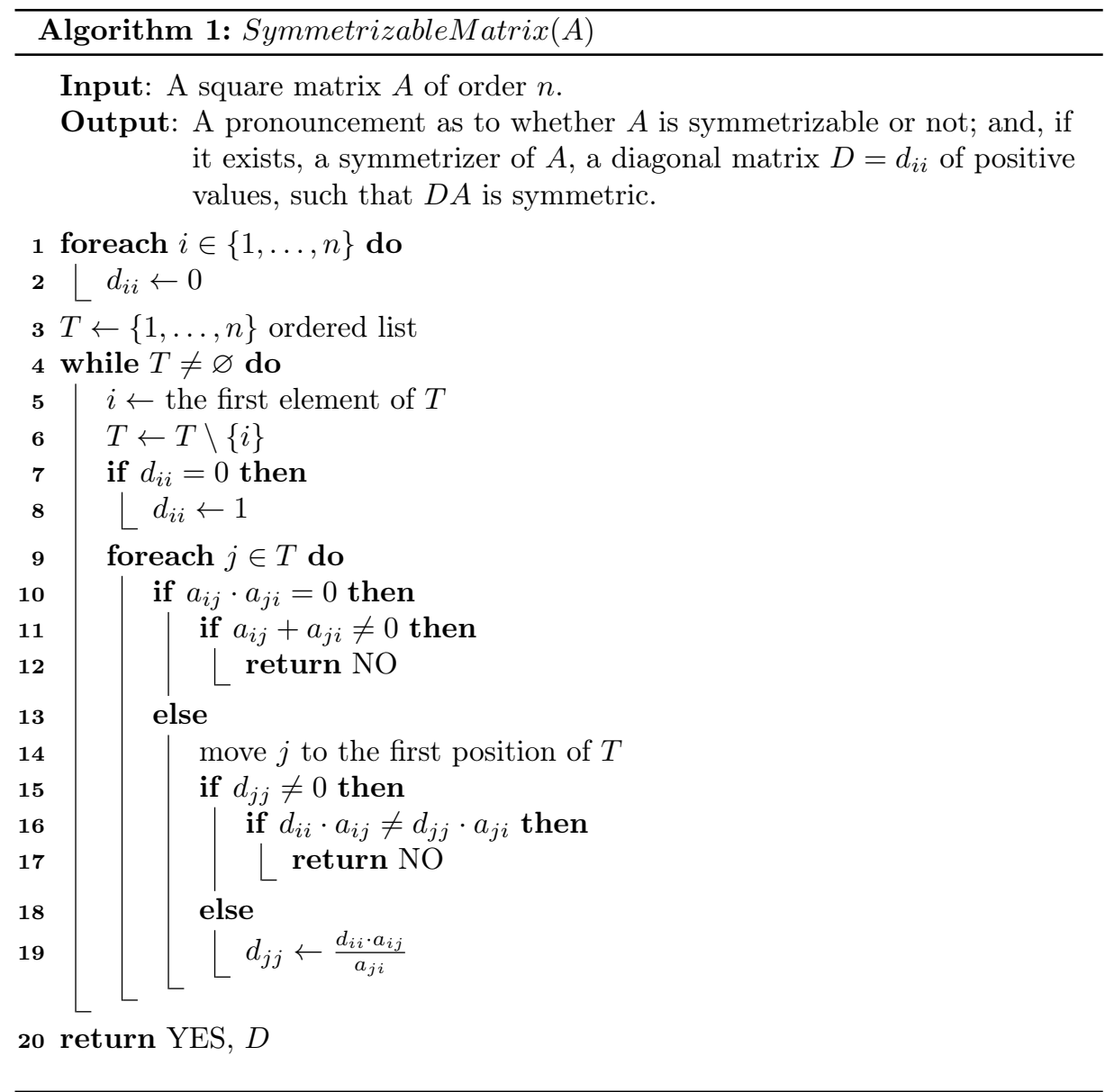

that $j \notin T$ will have been passed to the "while loop" without the return NO. Therefore, if the algorithm returns $D$, we have that $d_{i i} \cdot a_{i j}=d_{j j} \cdot a_{j i}$ for any pair of different parameters $i$ and $j$.

Suppose the algorithm returns NO when $A$ is symmetrizable. Since $A$ is symmetrizable, the algorithm returns NO on line 17 and therefore there exist $i$ and $j$ such that $d_{i i} \cdot a_{i j} \neq d_{j j} \cdot a_{j i}$.

Let $T$ be the list $\left\langle i_{1}, \ldots, i_{t}\right\rangle$ at the beginning of the "while loop". It follows from line 14, that the first indices $\star$ of $T$ have $d_{\star \star} \neq 0$ and the last ones have $d_{\star \star}=0$, that is there exists $k \in\{0, \ldots, t\}$ such that $d_{i_{s} i_{s}} \neq 0$ for all $1 \leq s \leq k$ and $d_{i_{s} i_{s}}=0$ for all $k<s \leq t$. Observe that $k=0$ implies that $d_{\star \star}=0$, for all $\star \in T$. Since $i$ is the first element in $T$ (line 5) and $d_{j j} \neq 0$ (line 15), we have that $d_{i i} \neq 0$ at the beginning of the "while loop". Therefore, $d_{i i}$ and $d_{j j}$ have been defined before and there exist $k \geq 3, i_{1}=i, i_{2}=j$ and $i_{3}, \ldots, i_{k} \notin T$ such that $a_{i_{1} i_{2}} \cdot a_{i_{2} i_{3}} \cdot \ldots \cdot a_{i_{k} i_{1}} \neq 0$. Since $A$ is symmetrizable, we have by Lemma 3.1, that $a_{i_{1} i_{2}} \cdot a_{i_{2} i_{3}} \cdot \ldots \cdot a_{i_{k} i_{1}}=a_{i_{2} i_{1}} \cdot a_{i_{3} i_{2}} \cdot \ldots \cdot a_{i_{1} i_{k}}$. This implies that $d_{i i} \cdot a_{i j}=d_{j j} \cdot a_{j i}$, a contradiction. 
Next algorithm does essentially the same as the above. The difference is that it does not verify if $A$ is symmetrizable but assume it and ends as soon as it have calculated all $d_{i i}$.

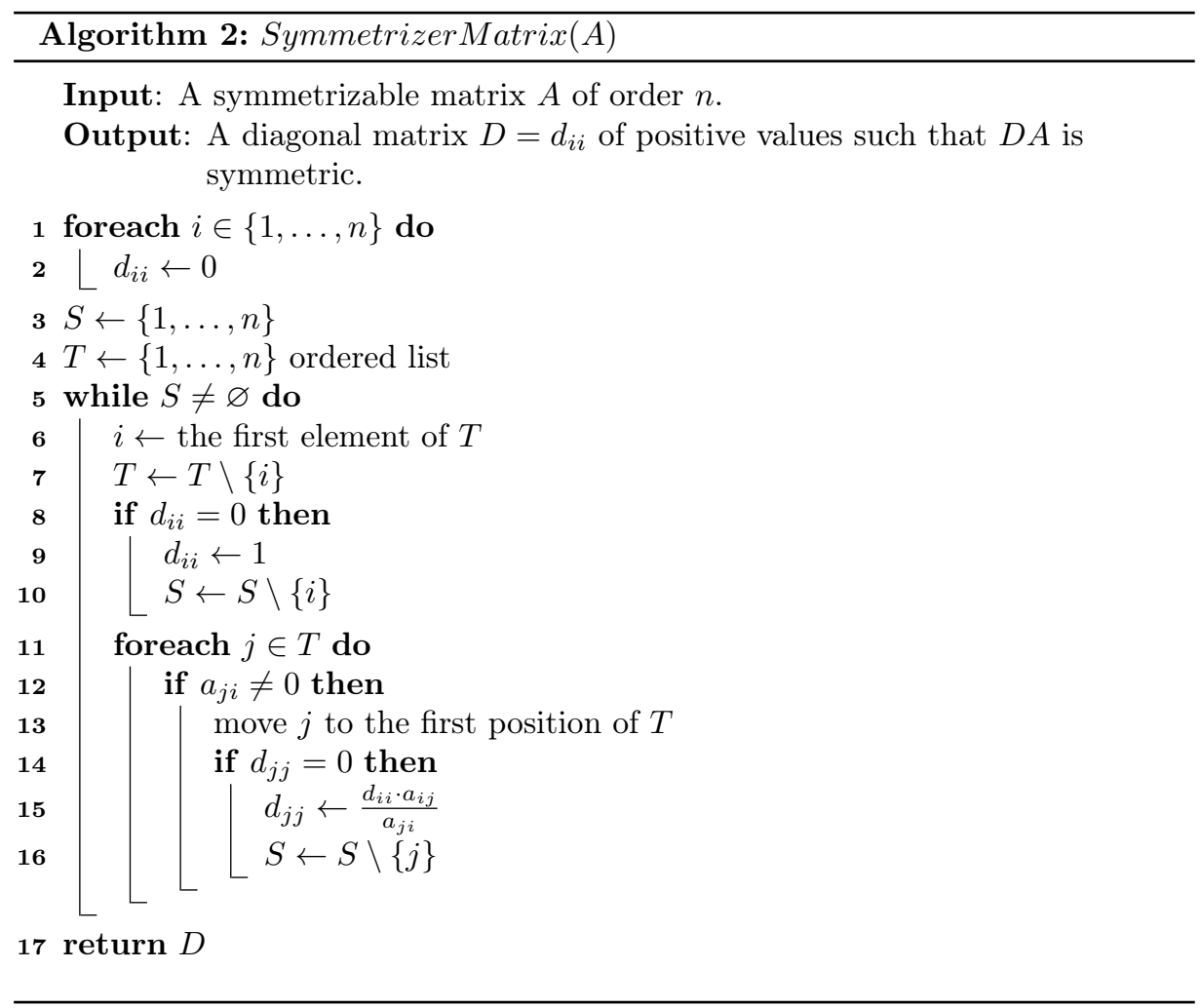

Proposition 3.2. Algorithm 2 is correct.

Proof. The behavior of Algorithm 2 is quite similar to that of Algorithm 1. The differences are that Algorithm 2 does not check whether $A$ is symmetrizable or not and the use of an additional list $S$ which maintains the elements $i$ such that $d_{i i}=0$ is not defined yet. Since the algorithm assumes that the input matrix $A$ is symmetrizable, this enables termination when all elements of $D$ have already been defined. This control is done by changing the condition of the "while loop" (line 5) accordingly and adding the operation to remove element $i$ from $S$ whenever $d_{i i}$ is defined (lines 10 and 16).

\section{POSITIVE QUASI-CARTAN COMPANION}

A symmetric matrix $A$ is positive definite if $x^{T} \cdot A \cdot x>0$ for all vectors $x$ of length $n$, with $x$ nonzero. If a symmetrized matrix $D C$ is positive definite, then we say that the quasi-Cartan matrix $C$ is positive. By Sylvester's criterion [3], being positive definite means that the principal minors of $D C$ are all positive. 
The following theorem is a generalization of Sylvester's criterion.

Theorem 4.1. Let $C$ be a symmetrizable matrix. The following conditions are equivalent:

(a) $C$ is positive.

(b) All principal minors of $C$ are positive.

(c) All leading principal minors of $C$ are positive.

Proof. Let $D$ be a symmetrizer matrix of $C$. Observe that $(D C)_{[i i]}=D_{[i i]} \times C_{[i i]}$. Thus, $\operatorname{det}\left((D C)_{[i i]}\right)=\operatorname{det}\left(D_{[i i]}\right) \times \operatorname{det}\left(C_{[i i]}\right)=d_{1} \cdot \ldots \cdot d_{i-1} \cdot d_{i+1} \ldots d_{n} \cdot \operatorname{det}\left(C_{[i i]}\right)$.

Since $d_{i}>0$ for all $i$, we have that $\operatorname{det}\left(C_{[i i]}\right)>0$ if and only if $\operatorname{det}\left((D C)_{[i i]}\right)>0$. Using induction, the result follows because principal matrices are obtained recursively in this way.

The following proposition shows that the property of having a positive quasi-Cartan companion is preserved for principal submatrices. This result leads intuitively to an inductive solution.

Proposition 4.1. If $B$ is a skew-symmetrizable matrix then $B$ has a positive quasi-Cartan companion matrix if and only if any principal submatrix of $B$ has a positive quasi-Cartan companion.

Proof. $\quad(\Rightarrow)$ Let $C$ be a positive quasi-Cartan companion of $B$. For induction, we need only that $C_{[i i]}$ is a symmetrizable matrix and therefore a positive quasi-Cartan companion of $B_{[i i]}$. Since $(D C)_{[i i]}=D_{[i i]} \times C_{[i i]}$ is symmetric, we have that $C_{[i i]}$ is a symmetrizable matrix. Similarly, $B_{[i i]}$ is skew-symmetrizable.

It follows from Theorem 4.1 that $C_{[i i]}$ is positive. Therefore, $C_{[i i]}$ is a positive quasi-Cartan companion of $B_{[i i]}$.

$(\Leftarrow)$ It follows from the fact that the matrix $B$ is a principal submatrix of itself.

Verifying all quasi-Cartan companions is exponential since if $\mathrm{B}$ is a skew-symme-trizable matrix of order $n$ then it has $\mathcal{O}\left(2^{n^{2}}\right)$ matrices $C$ which are quasi-Cartan companions of $B$. Note that a quasi-Cartan companion of $B$ is specified by choosing the signs of its off-diagonal matrix entries, with the only requirement being that $\operatorname{sgn}\left(c_{i j}\right)=\operatorname{sgn}\left(c_{j i}\right)$ and $\left|c_{i j}\right|=\left|b_{i j}\right|$ for all $i \neq j$.

From Theorem 4.1, one obtains a naive algorithm with time complexity $\Theta\left(n^{4}\right)$ to decide whether the given matrix $C$ is positive. One can also prove by induction that the Gaussian elimination can be directly applied to the symmetrizable matrix instead of its symmetrized matrix, which will leads to an algorithm with time complexity $\Theta\left(n^{3}\right)$.

The NP class consists of the decision problems for which a "yes" certificate can be checked in polynomial time. A decision problem $\pi$ is said to be NP-complete if every problem of NP can be polynomially reduced to $\pi$. For more information about polynomial reductions and the P, NP, 
and NP-complete classes, see $[5,10]$. Any of the algorithms presented above can be used as a verifier and, thus, we have proved that the problem of deciding whether there exists a positive quasi-Cartan companion belongs to NP. We also conjecture that this problem is NP-complete.

The following lemma gives a necessary condition for a quasi-Cartan companion to be positive. We present the main ideas of the original proof of this lemma, since they are useful in the sequel.

Lemma 4.1. (Barot, Geiss and Zelevinsky [1])

Let $C$ be a positive quasi-Cartan matrix. Then

(a) $0 \leq c_{i j} \cdot c_{j i} \leq 3$ for any $i$, $j$ such that $i \neq j$.

(b) $c_{i k} \cdot c_{k j} \cdot c_{j i}=c_{k i} \cdot c_{j k} \cdot c_{i j} \geq 0$ for any pairwise different $i, j, k$.

\section{Proof.}

(a) Let $C^{\prime}=\left(\begin{array}{cc}2 & c_{i j} \\ c_{j i} & 2\end{array}\right)$ be a principal submatrix of $C$. Since $C$ is a symmetrizable matrix, it is symmetric by signs and $c_{i j} \cdot c_{j i} \geq 0$. Since $C$ is positive, then $\operatorname{det}\left(C^{\prime}\right)=4-c_{i j} \cdot c_{j i}>$ 0 . Therefore, $c_{i j} \cdot c_{j i} \leq 3$.

Since $C$ is symmetrizable, we have that $\operatorname{sgn}\left(c_{i j}\right)=\operatorname{sgn}\left(c_{j i}\right)$.

(b) Let $c_{i k} \cdot c_{k j} \cdot c_{j i} \neq 0$. Since that $C$ is symmetrizable, from Lemma 3.1, one can see that $c_{k i} \cdot c_{j k} \cdot c_{i j}=c_{i k} \cdot c_{k j} \cdot c_{j i}$. The condition of positivity of the $3 \times 3$ principal minors of $C$ in the rows and columns $i, j, k$ can be rewritten as:

$$
c_{i k} \cdot c_{k j} \cdot c_{j i}>c_{i j} \cdot c_{j i}+c_{i k} \cdot c_{k i}+c_{j k} \cdot c_{k j}-4
$$

Since $c_{i k} \cdot c_{k j} \cdot c_{j i} \neq 0$ we have that $\left|c_{s t}\right| \geq 1$ and thus $c_{s t} \cdot c_{t s} \geq 1$ for $(s, t) \in$ $\{(i, k),(k, j),(j, i)\}$. Therefore, $c_{i k} \cdot c_{k j} \cdot c_{j i}>3-4=-1$. The result follows.

Condition (a) is obtained by studying submatrices of order 2 . We shall sharpen this limit by studying submatrices of order 3 . We observe that for a positive quasi-Cartan matrix of order 3 we have three leading principal submatrices:

(i) a submatrix of order 1 (that obviously has a positive determinant);

(ii) a submatrix of order 2 (that must be positive due Lemma 4.1, with $0 \leq c_{i j} \cdot c_{j i} \leq 3$ ); and, finally,

(iii) $C$ itself (which is the submatrix of order 3).

Definition 4.1. $C^{+}=\left(c_{i j}^{+}\right)$such that $c_{i j}^{+}=\left|b_{i j}\right|$ and $c_{i i}^{+}=2$.

Proposition 4.2. If $B$ is a skew-symmetrizable matrix of order 3 then $B$ has a positive quasiCartan companion if and only if the matrix $C^{+}=\left(c_{i j}^{+}\right)$is positive. 
Proof. $\quad \Rightarrow$ Suppose there exists a positive quasi-Cartan companion matrix $C$. Clearly, $0 \leq$ $c_{i j}^{+} \cdot c_{j i}^{+} \leq 3$. Thus, using Lemma 4.1,

$$
\begin{aligned}
\operatorname{det}\left(C^{+}\right) & =8-2 \cdot c_{j k}^{+} \cdot c_{k j}^{+}-2 \cdot c_{i j}^{+} \cdot c_{j i}^{+}-2 \cdot c_{k i}^{+} \cdot c_{i k}^{+}+2 \cdot c_{i j}^{+} \cdot c_{j k}^{+} \cdot c_{k i}^{+} \\
& =8-2 \cdot c_{j k} \cdot c_{k j}-2 \cdot c_{i j} \cdot c_{j i}-2 \cdot c_{k i} \cdot c_{i k}+2 \cdot\left|c_{i j} \cdot c_{j k} \cdot c_{k i}\right| \\
& \geq \operatorname{det}(C)>0 .
\end{aligned}
$$

Since all leading principal minors of $C^{+}$are positive, we have that $C^{+}$is also a positive quasiCartan companion of $B$.

$(\Leftarrow)$ Since $C$ is a quasi-Cartan matrix, it follows from Theorem 3.1 that $C^{+}$is a quasi-Cartan companion of $B$.

In the following results, we sharpen the bound in Lemma 4.1. Firstly, we examine the case $n=3$.

Lemma 4.2. Let $C$ be a positive quasi-Cartan matrix of order 3.

(a) If $C$ is connected, then $0 \leq c_{i j} \cdot c_{j i} \leq 2$ for any $i, j$ such that $i \neq j$.

(b) $0 \leq c_{12} \cdot c_{23} \cdot c_{31}=c_{13} \cdot c_{32} \cdot c_{21} \leq 2$.

Proof. Proposition 4.2 shows that $\operatorname{det}\left(C^{+}\right)>\operatorname{det}(C)$. Thus, $C^{+}$is positive. For the sake of simplicity, suppose that $C=C^{+}$.

(a) By Lemma 4.1, $0 \leq c_{i j} \cdot c_{j i} \leq 3$. Suppose, without loss of generality, that $c_{12}=3$. Then, $c_{21}=1$ and $\operatorname{det}(C)=8+2 \cdot c_{12} \cdot c_{23} \cdot c_{31}-2 \cdot c_{12} \cdot c_{21}-2 \cdot c_{13} \cdot c_{31}-2 \cdot c_{23} \cdot c_{32}=$ $2+6 \cdot c_{23} \cdot c_{31}-2 \cdot c_{13} \cdot c_{31}-2 \cdot c_{23} \cdot c_{32}>0$.

Since $C$ is connected, $c_{23} \neq 0$ or $c_{31} \neq 0$. The positivity of $C$ implies that both $c_{23}$ and $c_{31}$ are non zero. Recall that since $C$ is symmetrizable, we have that $c_{32} \neq 0$ and $c_{13} \neq 0$.

Since $c_{12} \cdot c_{23} \cdot c_{31}=c_{21} \cdot c_{32} \cdot c_{13}$, we have either that $3 \cdot c_{23} \cdot c_{31}=c_{32} \cdot c_{13}$ and $c_{32}=3$ or $c_{13}=3$. By symmetry, suppose that $c_{32}=3$. Then, $c_{23}=1$ and $c_{31}=c_{13}$. Since $\left(c_{13}\right)^{2}=c_{13} \cdot c_{31} \leq 3$, we conclude that $c_{31}=c_{13}=1$.

On the other hand, $\operatorname{det}(C)=2+6 \cdot c_{23} \cdot c_{31}-2 \cdot c_{13} \cdot c_{31}-2 \cdot c_{23} \cdot c_{32}=2+6-2-6=0$. This yields a contradiction to the positivity of $C$.

Therefore, $c_{i j} \cdot c_{j i} \leq 2$, for all $i, j$.

(b) Suppose that $c_{12} \cdot c_{23} \cdot c_{31}=c_{13} \cdot c_{32} \cdot c_{21} \geq 3$. If $c_{12} \cdot c_{21}=3$, using the contrapositive of (a) above, we have that $c_{23}=0$ and $c_{31}=0$. A contradiction to the hypothesis. This implies that $c_{12} \cdot c_{21} \leq 2$. In the same way, we obtain that $c_{i j} \cdot c_{j i} \leq 2$ for all $i, j$. Therefore, $c_{12} \cdot c_{23} \cdot c_{31} \geq 4$. We can suppose, without loss of generality, that $c_{12}=2$ and $c_{23}=2$. Thus, $c_{21}=1$ and $c_{32}=1$ and we obtain that $c_{13}=4$ and $c_{31} \neq 0$ a contradiction to Lemma 4.1. Therefore, $0 \leq c_{12} \cdot c_{23} \cdot c_{31} \leq 2$. 
We generalize Lemma 4.2 to account for any $n \geq 3$.

Theorem 4.2. Let $C$ be a positive quasi-Cartan matrix of order $n$, with $n \geq 3$.

(a) If $C$ is connected, then $0 \leq c_{i j} \cdot c_{j i} \leq 2$ for all $i, j$.

(b) $0 \leq c_{i k} \cdot c_{k j} \cdot c_{j i} \leq 2$ for all pairwise different $i, j, k$.

Proof. (a) Suppose that exist $i, j$ such that $c_{i j} \cdot c_{j i} \geq 3$. Since $n \geq 3$, there exists $k \notin\{i, j\}$. Consider the principal submatrix $C^{\prime}$ of $C$, consisting of the rows and columns $i, j$ and $k$. By Lemma 4.2, $C^{\prime}$ is disconnected. This implies that $c_{i k}=c_{k j}=0$ and thus $c_{k i}=c_{j k}=0$. Since this is true for all $k \notin\{i, j\}$, we have that $C$ is disconnected, a contradiction.

(b) It follows from Lemma 4.2 by considering the principal submatrix of $C$, consisting of the rows and columns $i, j$ and $k$.

As is shown in Theorem 4.3 below, when the skew-symmetrizable matrix has no zero entries, we only need to analyze the matrix $C^{+}$defined in 4.1 .

Theorem 4.3. If $B$ is a skew-symmetrizable matrix such that $b_{i j} \neq 0$, for $i \neq j$, then $B$ has $a$ positive quasi-Cartan companion if and only if $C^{+}=\left(c_{i j}^{+}\right)$defined by $c_{i j}^{+}=\left|b_{i j}\right|$ and $c_{i i}^{+}=2$ is positive.

Proof. $\quad(\Rightarrow)$ Suppose there exists a positive quasi-Cartan companion $C$. We shall show by induction that $C^{+}$is positive. If $C$ is $2 \times 2$ matrix, the result is clearly obtained. First, we show that $\operatorname{det}\left(C^{+}\right)>0$. Let $x_{i j}=\operatorname{sgn}\left(c_{i j}\right)$. Observe that $x_{j i}=x_{i j}$ and $x_{i i}=1$ due to it to being a quasi-Cartan matrix. Since $c_{i j} \neq 0$ there is no ambiguity in this definition. Define $x_{i}=x_{1 i}$ for all $i$. We shall show, by induction on $i$, that $x_{i j}=x_{i} \cdot x_{j}$ for all $i, j$. Clearly, $x_{i i}=\left(x_{i}\right)^{2}$.

Since $x_{1}=1$, we clearly have that $x_{1 i}=x_{1} \cdot x_{i}$. Suppose that $x_{k j}=x_{k} \cdot x_{j}$ for all $k<i$. By Theorem 4.2, $c_{i j} \cdot c_{j k} \cdot c_{k i}>0$. Thus, $x_{i j} \cdot x_{j k} \cdot x_{k i}=1$. Therefore, $x_{i j}=x_{j k} \cdot x_{k i}=x_{k j} \cdot x_{k i}=$ $x_{k} \cdot x_{j} \cdot x_{k} \cdot x_{i}=x_{i} \cdot x_{j}$.

Since $x_{i j}=x_{i} \cdot x_{j}$ and $c_{i j}=x_{i j} \cdot c_{i j}^{+}$, we have that $C=X C^{+} X$ where:

$$
X=\left(\begin{array}{ccc}
x_{1} & & 0 \\
& \ddots & \\
0 & & x_{n}
\end{array}\right)
$$

It follows that $\operatorname{det}(C)=\operatorname{det}(X) \cdot \operatorname{det}\left(C^{+}\right) \cdot \operatorname{det}(X)=\operatorname{det}\left(C^{+}\right)$. By induction on the dimension of $C^{+}$, we have that all leading principal minors of $C^{+}$are positive.

$(\Leftarrow)$ It follows from the fact that $C$ is quasi-Cartan matrix and by Theorem 3.1 , that $C^{+}$is a quasi-Cartan companion of $B$. 
We observe that Theorem 4.3 does not hold for all skew-symmetrizable matrices, as we can be seen in the following example:

Let $B=\left(\begin{array}{cccc}0 & 1 & 1 & 0 \\ 1 & 0 & 0 & 1 \\ 1 & 0 & 0 & 1 \\ 0 & 1 & 1 & 0\end{array}\right), \quad C=\left(\begin{array}{cccc}2 & -1 & 1 & 0 \\ -1 & 2 & 0 & 1 \\ 1 & 0 & 2 & 1 \\ 0 & 1 & 1 & 2\end{array}\right), \quad C^{+}=\left(\begin{array}{llll}2 & 1 & 1 & 0 \\ 1 & 2 & 0 & 1 \\ 1 & 0 & 2 & 1 \\ 0 & 1 & 1 & 2\end{array}\right)$.

The quasi-Cartan companion $C^{+}$is not positive, but $C$ is positive.

The following case always has a positive quasi-Cartan companion.

Proposition 4.3. If $B$ is the skew-symmetric matrix defined by $\left|b_{i j}\right|=1$ for all $i \neq j$, then $B$ has a positive quasi-Cartan companion.

Proof. If $B$ is a matrix of order $n$, then we can calculate that $\operatorname{det}\left(C^{+}\right)=n+1$. Clearly, $C^{+}$is positive. The result follows from Theorem 4.3.

\section{CONCLUSIONS}

In this paper, we report some theoretical advances for the problem of deciding upon the existence of a positive quasi-Cartan companion for skew-symmetrizable matrices. We show that this decision problem is in NP and conjecture that it is NP-complete.

\section{ACKNOWLEDGMENTS}

We would like to thank CAPES, CNPq, FAPEG and FAPERJ for their partial support received while this research was conducted. Also, the authors would like to thank the professor Leslie Richard Foulds, from Universidade Federal de Goiás, that gave valuable suggestions to improve the paper.

RESUMO. Grafos são comumente representados por matrizes que também são usadas para representar álgebras e álgebras cluster. Este artigo mostra algumas propriedades de matrizes, a fim de facilitar a compreensão das matrizes simetrizáveis com características específicas, chamadas matrizes companheiras quase-Cartan posi-tivas, e o problema de localizá-las. Neste contexto, matrizes simetrizáveis são aquelas que são simétricas quando multiplicadas por uma matriz diagonal com entradas positivas chamada matriz simetrizante. Conjecturamos que este problema é NP-completo e nós mostramos que ele está em NP generalizando o critério de Sylvester para matrizes simetrizáveis. Nós estreitamos os limites dos coeficientes conhecidos para tais matrizes.

Palavras-chave: matriz simetrizável, companheira quase-Cartan, algoritmo. 


\section{REFERENCES}

[1] M. Barot, C. Geiss \& A. Zelevinsky. Cluster algebras of finite type and positive symmetrizable matrices. J. London Math. Soc., 73 (2006), 545-564.

[2] E. Cartan. Les groupes de transformations continus, infinis, simples. Ann. Sci. École Norm. Sup., 26 (1909), 93-161.

[3] W-K. Chen. "Theory and Design of Broadband Matching Networks", Pergamon Press Ltd., Headington Hill Hall, Oxford, UK, (1976).

[4] C.J. Colbourn \& B.D. McKay. A corretion to Colbourn's paper on the complexity of matrix symmetrizability. Inform. Proc. Letters, 11 (1980), 96-97.

[5] T.H. Cormen, C.E. Leiserson, C. Stein \& R.L. Rivest. "Introduction to Algorithms". The MIT Press, Cambridge, MA, USA, 2nd edition, (2002).

[6] S. Fomin \& A. Zelevinsky. Cluster algebras I: Foundations. J. Amer. Math. Soc., 15(2) (2002), 497-529 (online).

[7] S. Fomin \& A. Zelevinsky. Cluster algebras II: finite type classification. Invent. Math., 154 (2003), 63-121.

[8] W. Killing. Die rechnung in den nicht-euklidischen raumformen. J. Reine Angew. Math., 89 (1880), 265-287.

[9] E.L. Lima. “Álgebra Linear”, Associação Instituto Nacional de Matemática Pura e Aplicada, Rio de Janeiro, Coleção Matemática Universitária, 6th edition, (2003).

[10] M. Sipser. "Introduction to the Theory of Computation". Thomson Press, Faridabad, Haryana, India, 2nd edition, (2006). 